\title{
K-RAS and P53 mutations in association with COX-2 and hTERT expression and clinico-pathological status of NSCLC patients
}

\author{
Mojca Stražišar, Tomaž Rott and Damjan Glavač* \\ Department of Molecular Genetics, Faculty of Medicine, University of Ljubljana, Ljubljana, Slovenia
}

\begin{abstract}
We evaluated the occurrence of mutations in P53, K-RAS, COX-2, expression of COX-2 and hTERT and relations among clinicopathological signs. P53 mutations were identified in $34.4 \%$ of tumours, the majority of them occurring in SCC (squamous cell carcinoma, 55.6\%). K-RAS was mutated in $12.2 \%$ of NSCLC tumours, the majority of the mutations being found in ADC (adenocarcinoma, 27.0\%). Mutational screening detected three different COX-2 mutations and five different P53 mutations, published for the first time. With RT-PCR we observed that the expression of the tested genes, hTERT and COX-2, was highly significant for ADC $(p<0.01)$ and SCC $(p<0.05)$. Statistical analysis of the combined results revealed significant correlation between expression of COX-2 and hTERT $(p<0.001)$, hTERT expression and staging $(p<0.05)$ and survival $(p<$ 0.01). A positive correlation between COX-2 expression and K-RAS mutation $(p<0.05)$ was also observed. This study provides insight into associations between the analysed biomarkers and the clinical-pathological data of the patients.
\end{abstract}

Keywords: NSCLC, P53, K-RAS, hTERT, COX-2, mutation, expression

\section{Introduction}

Non-small-cell lung cancer (NSCLC) includes adenocarcinoma (ADC), squamous cell carcinoma (SCC) and large cell carcinoma (LCC), types of lung cancer with similar clinical and biological characteristics [1,2]. Different types of NSCLC are the result of carcinogenesis in different types of cells, which undergo stepwise malignant progression because of the accumulation of genetic alterations [2].

Various genes that undergo genetic alterations in lung cancer cells have so far been identified. The most frequently described mutated genes are P53 (alterations have been detected in $\sim 50 \%$ of NSCLC) and K-RAS (alterations have been detected in $\sim 15 \%$ of

\footnotetext{
*Corresponding author: Damjan Glavač, Ph.D., Associate Professor of Human Genetics, Department of Molecular Genetics, Faculty of Medicine, Institute of Pathology, Korytkova 2, 1000 Ljubljana, Slovenia. Tel.: +386 15437180, Fax: +386 15437181; E-mail: damjan.glavac@mf.uni-lj.si.
}

NSCLC) [2]. Defects in P53 are common in NSCLC, suggesting that this pathway is essential for the regulation of growth, differentiation and death of lung epithelial cells $[1,3]$. Alterations of the RAS genes (mutations occur mainly on K-RAS and account for $\sim 90 \%$ of RAS mutations in NSCLC, predominantly in ADC) play an essential role in signal transduction instability and can consecutively cooperate in lung tumourigenesis $[2,4]$. It has generally been accepted that mutations in either P53 or K-RAS or both may be of prognostic significance in early stage NSCLC [5,6].

The search for prognostic factors in NSCLC has expanded over the years from mutation detection to gene expression detection. Telomerase can be expressed in up to $85 \%$ of NSCLC and thus plays a critical role in maintaining cellular immortality [7-9]. Positive hTERT expression has been significantly associated with worse survival [7-9]. It has also been published that a combination of P53 mutation and telomerase over-expression may induce tumourigenesis in NSCLC [10,11]. There is multiple evidence of elevated 
COX-2 levels in NSCLC and their importance in lung carcinogenesis [12-14]. Over-expression of COX-2 has been proposed as a biomarker for biologically aggressive types of NSCLC and poorer survival [15-17]. Some publications have statistically evaluated the combination of more than one prognostic factor for gaining higher statistical power and/or researching the correlation between two or more independent factors. It has been shown that combined analysis of COX-2 and P53 expression or P53 gene mutation and telomerase activity may be useful for identifying the severity of NSCLC and the patient's prognosis $[10,18]$.

In this study, we examined possible relationships between somatic mutations in P53, K-RAS and COX-2 and expression levels in COX-2 and hTERT, in order to investigate the specific associations between these alterations and clinical data in NSCLC overall and in all three types of NSCLC separately.

\section{Material and methods}

\subsection{Patients}

The examined NSCLC patient group consisted of 75 $(83.3 \%)$ males and $15(16.7 \%)$ females, with ages ranging from 48 to 83 years (mean \pm standard deviation; $65.4 \pm 9.1$ years, data shown in Table 1). Seventyeight out of 90 patients stated that they were smokers $(86.7 \%)$, of which 67 were male $(89.3 \%)$ and 11 female (73.3\%). The median follow-up of the patients was 8.9 years (from 7.7 to 14.8 years). Patients who died during the study were considered to have been followed until the end of the study. To our knowledge, patients were not chronically subjected to any other environmental exposure known to promote the development of lung cancer (uranium or coal mines; asbestos, mustard gas or radon exposure). The National Medical Ethics Committee of the Republic of Slovenia (NMEC) approved the study. Our analysis included 90 surgical specimens of patients with primary non-small cell lung carcinoma (34 adenocarcinoma, 45 squamous cell carcinoma, 11 large cell carcinoma, data shown in detail in Table 1). Lung resectates were examined by an expert pathologist. Adjacent macroscopically normal lung tissue was obtained several $\mathrm{cm}$ from the tumour whenever possible and used as a control. All samples were snap-frozen in liquid nitrogen and stored at $-80^{\circ} \mathrm{C}$ until RNA and DNA isolation.

\subsection{DNA extraction and mutation detection}

DNA was extracted from snap frozen lung cancer tumours and corresponding normal tissue using NucPrep $^{\mathrm{TM}}$ chemistry (Applied Biosystems, Foster city, CA, USA) on ABI 6100 (Applied Biosystems, Foster city, CA, USA). P53, K-RAS and COX-2 mutational status was determined by denaturising highpressure liquid chromatography (DHPLC) mutation analysis (Transgenomic, USA). For DHPLC of the P53 gene, primers and conditions were used as described previously [19]. For DHPLC screening of K-RAS mutational hotspots in exons one and two, primers published by Konig et al. were used [20] and column temperature was calculated with Wavemaker ${ }^{\mathrm{TM}}$.

Newly designed primers (Table 2), suitable for $\mathrm{DH}-$ PLC mutation detection for COX-2 gene, were chosen with an online oligo-designer (IDT ${ }^{\circledR}$ SCITools Oligo Design and Analysis), and the conditions for DHPLC mutation screening were selected according to Wavemaker ${ }^{\mathrm{TM}}$ (Transgenomic, USA).

\subsection{RNA extraction, $c D N A$ synthesis and gene expression detection}

RNA for the expression analysis was extracted with TRIzol ${ }^{\circledR}$ reagent and chloroform. After extraction, total RNA was cleaned with Purelink ${ }^{\text {TM }}$ Micro-toMidi System (Invitrogen,ZDA). First strand cDNA was synthesized using MultiScribe ${ }^{\mathrm{TM}}$ reverse transcriptase (Applied BioSystems, Foster City, California, USA), with random hexamers, according to the manufacturer's instructions.

Expression of the COX-2 and hTERT genes in tumours relative to their normal adjacent tissues was measured using quantitative real-time PCR (RT-PCR) based on the TaqMan ${ }^{\circledR}$ fluorescence methodology. A ready mixture of probes and primers specific for COX-2 (Assay-on-Demand ${ }^{\mathrm{TM}}$, Hs0015133_m1; Applied Biosystems, Foster City, USA) and hTERT (Assay-on-Demand $^{\mathrm{TM}}$, Hs99999022_m1; Applied Biosystems, Foster City, USA) expression and human 18S rRNA as the endogenous control gene were used (TaqMan pre-developed assay reagents for gene expression; Applied BioSystems). The relative quantification of mRNA levels of the target genes (quantity of transcripts of the target in tumours relative to normal tissues) was determined using the $\Delta \Delta \mathrm{C}_{\mathrm{T}}\left(\Delta \mathrm{C}_{\mathrm{T}}\right.$ tumour- $\Delta \mathrm{C}_{\mathrm{T}}$ normal) method. If $\Delta \Delta \mathrm{C}_{\mathrm{T}}$ was significantly higher or lower than zero, the expression was considered to be statistically significantly over or under expressed. 
Table 1

Data showing histological (locations of the tumours, pTNM) and clinical (survival, age at the diagnosis, gender distribution) data of patients whose tumour samples were used in this study (NSCLC - non-small cell lung cancer, ADC - adenocarcinoma, LCC - large cell carcinoma, SCC - squamous cell lung carcinoma, vs. - versus, ${ }^{*}$ classified according to the American Joint Committee for Cancer Staging System)

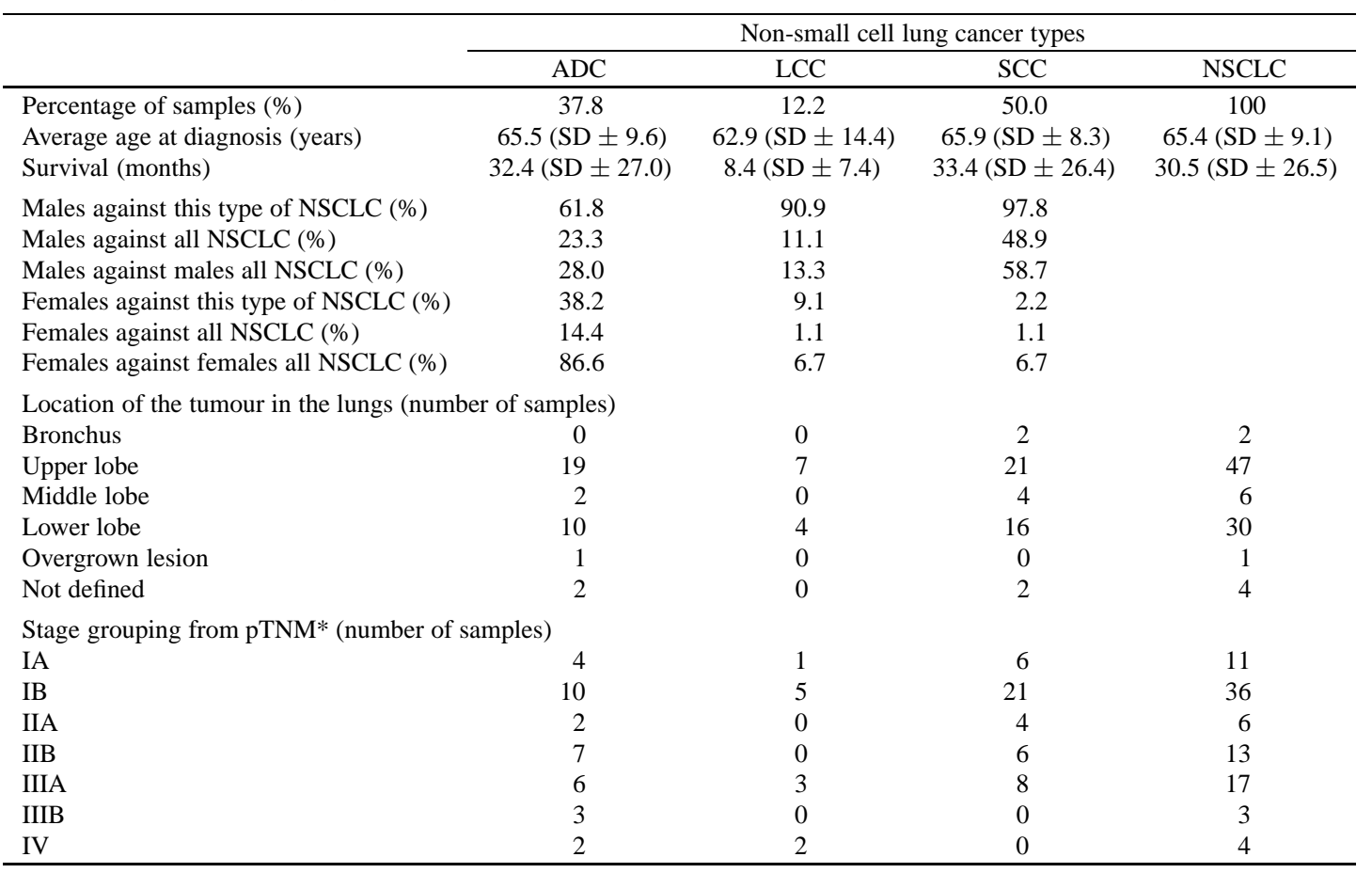

Table 2

Primers used for genomic DHPLC analysis, oligonucleotide sequences of PCR primers, PCR product lengths and annealing temperatures for COX-2 gene

\begin{tabular}{lllcc}
\hline Exon & 5' Forward primers & 5' Reverse primers & Length $(\mathrm{bp})$ & Annealing $\mathrm{T}\left({ }^{\circ} \mathrm{C}\right)$ \\
\hline EX1+UTR & AGGCGGAAAGAAACAGTCA & GGAGGTCAGAGCGGAAACT & 336 & 57 \\
EX2+3 & TGATTTATTTGCTGTCCT & CTGTATAAACTGGATGGG & 593 & 48 \\
EX4 & CTCAGGTGCCACTTTCCA & CAATGCAGCCCGTCTTAT & 293 & 50 \\
EX5 & GATTCTTACCTTAGCTTTCT & TAATGTTAGCCCTTGACT & 278 & 48 \\
EX6+7 & AAACTTCAACAGCAACAA & TTTTCATTTACCACATCT & 596 & 47 \\
EX8 & ATTGCATTCCAGTTGCTT & GTCTTTTGTTTTGGTTTCA & 394 & 48 \\
EX9 & CCTAAGGCAAGCTGAATA & GAAGTTGAGGCAGGAGAA & 398 & 50 \\
EX10 & CTGTCTTCATCGCCTTCA & GACTCCTTTCTCCGCAAC & 571 & 51 \\
\hline
\end{tabular}

\subsection{Statistics}

Survival was examined using the Kaplan-Meier method and variable effects on survival time were analyzed using Cox's regression model. Magnitudes and directions of the associations between intervals or ratio scale variables were determined using Spearman's Rho non-parametric test and comparisons between two independent samples with the Mann-Whitney U test, as appropriate. We used the Chi-square test for determining the association between mutations in K-RAS and P53 and ESE motifs. Differences were considered significant at $p<0.05$. For statistical evaluation of the alteration abundances, we used the Fisher's exact test. All statistical analyses were performed using SPSS version 14 (SPSS Inc., Illinois).

\section{Results}

\subsection{Disease outcome}

Survival of the patients was examined using the Kaplan-Meier method and survival differences were analyzed using the log-rank (Mantel-Cox) test. Tests of equality of survival distributions for the different 


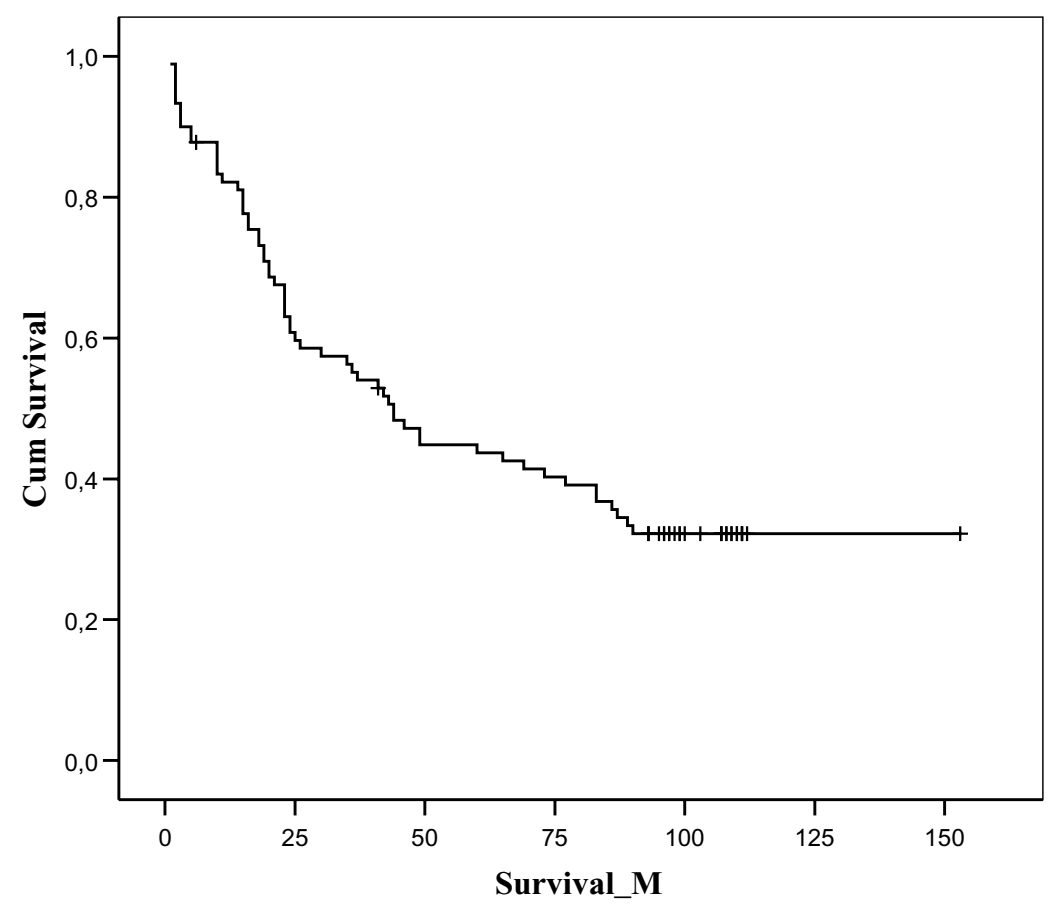

Fig. 1. Cumulative survival rate of patients with NSCLC whose samples were used in this study (survival $\mathrm{M}-$ survival in months, +- censored data).

cancer types (Chi $=0.351$ and $p=0.839)$ and tumour location $(\mathrm{Chi}=2.655, p=0.753)$ were not statistically significant (Figs 1 and 2).

Elevated expression of COX-2 was observed in $60.0 \%$ of tested NSCLC $(61.8 \%$ in ADC, $27.3 \%$ in LCC and $66.7 \%$ in SCC, Table 3 ) and hTERT in $55.6 \%$ (61.7\% in ADC, $27.3 \%$ in LCC and $57.8 \%$ in SCC). Average overall $\Delta \Delta \mathrm{C}_{\mathrm{T}}$ for COX-2 in NSCLC was 5.3 $(\mathrm{SD} \pm 0.6)$ and average overall $\Delta \Delta \mathrm{C}_{\mathrm{T}}$ for hTERT 8.2 $(\mathrm{SD} \pm 0.9)$.

Using Cox's regression model with multiple variables, no statistically significant association between survival and observed expression in COX-2 $(p=0.187)$ and in hTERT ( $p=0.552$ ) was found. There was also no statistically significant association between mutation in K-RAS ( $p=0.554)$ or P53 ( $p=0.963)$ and patient survival.

\subsection{Mutational screening}

We detected 36 P53 alterations in 31 NSCLC tumours (one ADC and two SCC samples with 2 alterations), 5 of them to our knowledge published for the first time; 470_490 del (V157fs), 757_758delAC (T253fs), 875_919 del + del 25 (K292fs), S362N (AGC $>$ AAC), 1056-1100del + del 14(D352fs). Three
(8.3\% NSCLC) of the detected mutations were silent; all others resulted in a change of amino acid $(63.9 \%$ NSCLC), or in frame shift or abbreviation of the p53 protein (27.0\% NSCLC). Twenty-five percent of the mutations are transitions and $50.0 \%$ transversions, $55.6 \%$ of all transversions are $\mathrm{G}>\mathrm{T}$ changes (Table 4). Codon V157 is the target of the mutation 4 times, codons G245, R283 three times and codon R273 twice (Table 4). Of all frame shift mutations (22.2\% of all mutations found in P53), $62.5 \%$ result in a premature stop codon. We also found one $\mathrm{G}>\mathrm{T}$ transversion, which causes an E204X change (Table 4). Mutations causing a premature stop of translation are K139fsX169, H178fsX180, E204X, N239fsX239, T253fsX262, E285fsX304 and P301fsX305. Four different mutations were found in two and mutation R283P in 3 tumours (Table 4). P53 was mutated in $26.5 \%$ ADC, $27.3 \%$ LCC and $55.6 \%$ SCC. In terms of frequencies of mutations found in P53, 69.4\% of all mutations were found in SCC (Table 5). The frequency of P53 mutation was significantly different between SCC and ADC (Fisher's exact test, $p=0.0122$ ).

K-RAS mutations were found in 13 NSCLC tumours; all had been previously described at codon hotspots (codons: 12 (76.9\%), $13(15.4 \%)$ and 61 (7.7\%)) and all were missense (Table 4$)$. Of all mutations, 
Table 3

Average overall results $\Delta \Delta \mathrm{C}_{\mathrm{T}}$ for expression levels of COX-2 and hTERT genes for different types of NSCLC. (COX-2 and hTERT expression results refer to the relative quantification of mRNA levels of the target genes. Results are the quantity of transcripts of COX-2 and hTERT mRNAs in tumour samples relative to normal tissues, determined using the $\Delta \Delta \mathrm{C}_{\mathrm{T}}\left(\Delta \mathrm{C}_{\mathrm{T}}\right.$ tumour $-\Delta \mathrm{C}_{\mathrm{T}}$ normal $)$ method; $\Delta \Delta \mathrm{C}_{\mathrm{T}}$; relative comparative cycle threshold number for quantification of mRNA levels of the target genes). The expression of COX-2 and hTERT was higher in all tumours regardless of the NSCLC type

\begin{tabular}{lccc}
\hline Lung cancer type & ADC & LCC & SCC \\
\hline Average overall $\mathrm{C}_{T} \mathrm{COX}-2$ & $8.9(\mathrm{SD} \pm 0.9)$ & $2.3(\mathrm{SD} \pm 0.1)$ & $3.0(\mathrm{SD} \pm 0.2)$ \\
Average overall $\mathrm{C}_{T}$ of hTERT & $12.1(\mathrm{SD} \pm 1.0)$ & $5.6(\mathrm{SD} \pm 0.3)$ & $5.3(\mathrm{SD} \pm 0.2)$ \\
\hline
\end{tabular}

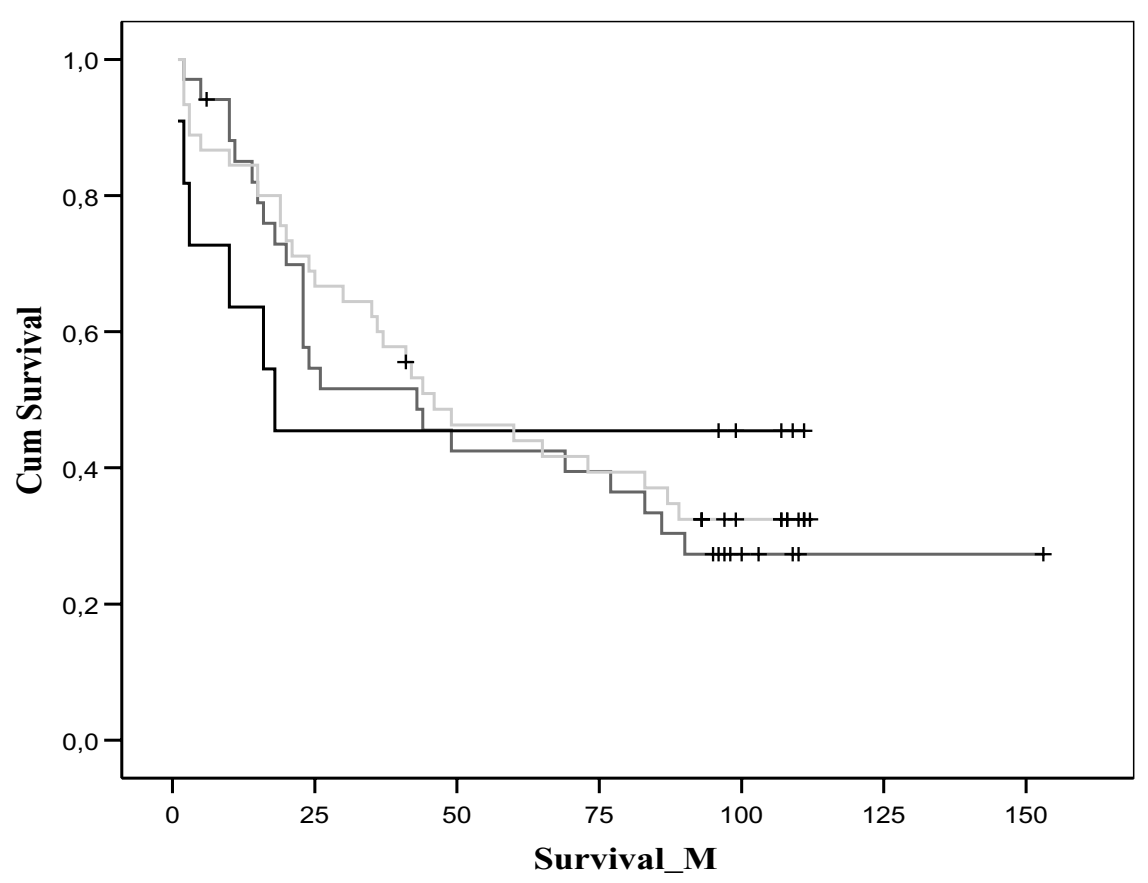

Fig. 2. Cumulative survival rate of patients with different types of NSCLC whose samples were used in this study (black - LCC, dark grey ADC, light gray - SCC; survival_M - survival in months, + - censored data).

$23.1 \%$ were $\mathrm{G}>\mathrm{T}$ transversions. $\mathrm{G} 12 \mathrm{~A}$ was detected in two ADC and in one LCC sample. G12R and G12D were detected in two samples. K-RAS was mutated in $27.0 \% \mathrm{ADC}, 18.2 \% \mathrm{LCC}$ and in $2.2 \%$ SCC. In terms of frequencies of mutations, we found $76.9 \%$ of all mutations in K-RAS in ADC (Table 5). In 12 cases, the affected aminoacid was glycine and in one case glutamine (Table 4). Fisher's exact test showed a statistically significant difference in frequency of K-RAS mutations between ADC and SCC $(p=0.035)$.

Mutation detection in the COX-2 gene resulted in the detection of 3 different alterations not to our knowledge previously published. V102V (GTG $>$ GTC) was found in SCC, H212H (CAT>CAC) in ADC and P593L $(\mathrm{CCC}>\mathrm{CTC})$ in two LCC tumours. Both patients with those mutations were male and their survival was less than 2 months. All detected alterations were heterozygous and detected only in tumours. The distribution of overall mutations showed that the majority of mutations were found in SCC (Table 5), although there are differences in the frequencies of mutations in different types of NSCLC.

\subsection{Comparisons between different parameters}

For statistical evaluation of the relationship between hTERT and COX-2 expression, patient survival, staging, age at diagnosis in overall NSCLC patients, we used Spearman's Rho test statistic. A significant positive correlation was found between expression of COX-2 and hTERT $(p<0.001)$ and hTERT expres- 
Table 4

Mutations found in P53 and K-RAS genes in non-small cell lung tumours according to the type and position. (No. of mutations - number of different types of mutations, and percentage of all mutations found in P53 or K-RAS genes; Other* - inframe deletion of three base pairs; V157-3 missense mutations resulting in change of aminoacid, and one deletion of the codon; R273 - 2 SCC tumours with R273H mutation, R283 - mutation $\mathrm{R} 283 \mathrm{P}$ was detected in one ADC, one LCC and one SCC)

\begin{tabular}{lclc}
\hline Mutations in P53 & No. of mutations & Mutations in K-RAS & No. of mutations \\
\hline Transitions & $9(25.0 \%)$ & Transitions & $3(23.1 \%)$ \\
Transversions & $18(50.0 \%)$ & Transversions & $10(76.9 \%)$ \\
Other & $9(25.0 \%)$ & Other & 0 \\
SUM & $36(100 \%)$ & SUM & $13(100 \%)$ \\
Silent mutations & $3(8.3 \%)$ & Silent mutations & 0 \\
Missense mutations & $23(63.9 \%)$ & Missense mutations & $13(100 \%)$ \\
Other* & $1(2.8 \%)$ & Other* & 0 \\
Change in RF & $8(22.2 \%)$ & Change in RF & 0 \\
Nonsense & $1(2.8 \%)$ & Nonsense & 0 \\
SUM & $36(100 \%)$ & SUM & $13(100 \%)$ \\
V157 & $4(11.1 \%)$ & G12 & $10(76.9 \%)$ \\
G245 & $3(8.3 \%)$ & G13 & $2(15.4 \%)$ \\
R273 & $2(5.6 \%)$ & Q61 & $1(7.7 \%)$ \\
R283 & $3(8.3 \%)$ & & \\
Other mutations & $24(66.7 \%)$ & Other mutations & 0 \\
SUM & $36(100 \%)$ & SUM & $13(100 \%)$ \\
\hline
\end{tabular}

Table 5

Distribution of mutations in P53, K-RAS and COX-2, detected in squamous carcinoma, adenocarcinoma and large cell carcinoma. Percentages show fractions of the mutations in one type of cancer versus the total mutations found in a particular gene (SCC - squamous cell carcinoma, ADC - adenocarcinoma, LCC - large cell carcinoma, NSCLC - non-small cell lung cancer)

\begin{tabular}{lcrrr}
\hline Mutations found & SCC & \multicolumn{1}{c}{ ADC } & \multicolumn{1}{c}{ LCC } & \multicolumn{1}{c}{ NSCLC } \\
\hline P53 & $25(69.4 \%)$ & $8(22.2 \%)$ & $3(8.4 \%)$ & $36(100 \%)$ \\
K-RAS & $1(7.7 \%)$ & $10(76.9 \%)$ & $2(15.4 \%)$ & $13(100 \%)$ \\
COX-2 & $1(25.0 \%)$ & $1(25.0 \%)$ & $2(50.0 \%)$ & $4(100 \%)$ \\
All & $27(50.9 \%)$ & $19(35.8 \%)$ & $7(13.2 \%)$ & $53(100 \%)$ \\
\hline
\end{tabular}

sion and staging $(p<0.05)$. A significant negative correlation was found between hTERT expression and survival $(p<0.01)$. We also determined that although staging showed no correlation with survival $(p=0.05)$, $\mathrm{pN}$ was negatively correlated with survival $(p<0.01)$ and $\mathrm{pM}$ with age at diagnosis $(p<0.05)$.

The Mann-Whitney test (exact 2-tailed $\sigma$ ) for investigating possible differences between groups with mutations in P53 and/or K-RAS and between different cancer types was used. COX-2 expression was highly significant in $\operatorname{ADC}(p<0.01)$ and in SCC $(p<0.01)$. hTERT expression was also highly significant in ADC $(p<0.01)$ and SCC $(p<0.05)$. Comparisons between groups with P53 and K-RAS showed positive correlation between COX-2 expression and K-RAS mutations $(p<0.05$; Fig. 3). The Mann-Whitney test was also performed for differentiation between genders. hTERT and COX-2 expression was found to be significantly higher in females $(p<0.05$, Fig. 4$)$.

\section{Discussion}

We examined the relationships between somatic mutations in P53 and K-RAS and expression of COX-2 and hTERT, in order to investigate the specific associations between these alterations and clinical data (survival, age at diagnosis, histology, staging) in NSCLC overall and in all three types of NSCLC independently. Each marker has already been studied as a prognostic factor based on the roles in carcinogenesis and frequencies of alterations found in lung cancer [12,15, 21]. There are also published studies of the association between two or more of the above studied markers [18, 22-24]. We analysed 90 patients with complete information for four biomarkers and histological and clinical data. Our major findings are as follows. We could not predict survival or test the variables with the COX regression model with clinicopathological data (staging, type of lung cancer, location of tumour, gender 


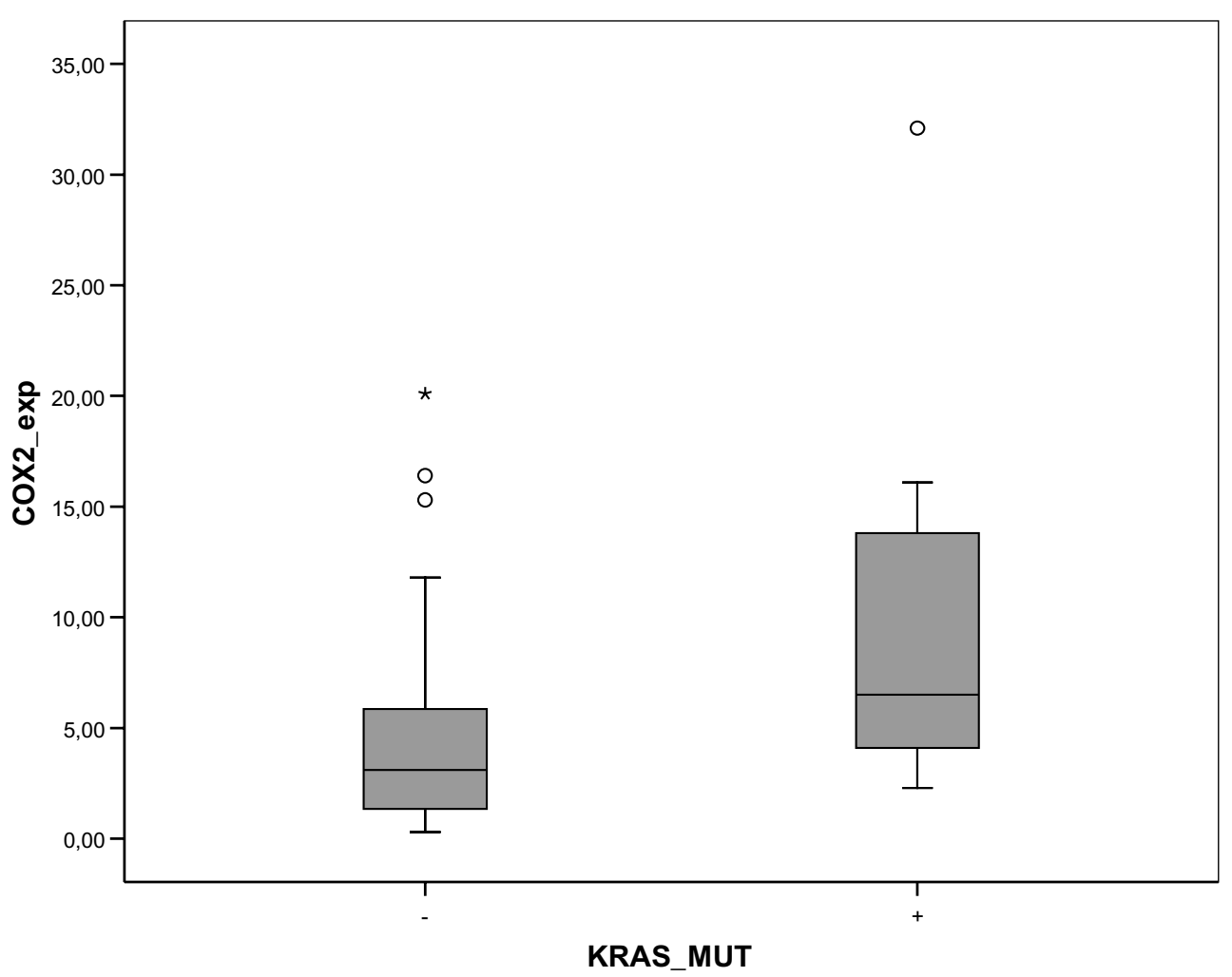

Fig. 3. Box-plot chart of the association of COX-2 expression and K-RAS mutation, within the NSCLC group (+ - group of NSCLC tumours with K-RAS mutation, - - group of NSCLC tumours without mutation). It shows that K-RAS mutations positively correlate with higher COX-2 expression.

and age at diagnosis) and the four analysed biomarkers. Patient survival was also examined using the KaplanMeier method and survival differences were analyzed using the log-rank (Mantel-Cox) test; distributions for the different cancer types and tumour location were not statistically significant. The lack of significance could be due to the small number of patients with a specific type of lung cancer (for example 11 patients with large cell carcinoma) or the overall number of patients $(90$ patients were included in the study), although the absence of prognostic significance of NSCLC when testing different variables with the COX proportional model has already been published on a much larger patient group [23]. One possibility is that variables and clinicopathological data together are less informative for prediction of survival but they could still be associated, so we used two other appropriate test statistics for determination of the association.

The results of Spearman's test showed a positive correlation between COX-2 and hTERT expression, as already described by Lu et al. [22], and between hTERT and staging. We also proved a negative correlation between survival and hTERT expression. There is also an interesting result concerning staging and testing $\mathrm{pT}$, $\mathrm{pN}$ and $\mathrm{pM}$ independently. Although staging showed no correlation with survival and age at diagnosis, if tested separately and independently, $\mathrm{pN}$ correlated with worse survival and pM with earlier age at diagnosis. pT in our case showed no significant correlation with survival and age at diagnosis.

Mutation screening of the P53 and K-RAS revealed high mutation frequencies. The frequency of P53 mutation in different NSCLC types was significant only for squamous cell carcinoma. The mutational pattern was very heterogeneous, mutations occurred at 29 different codons and only 4 mutations occurred more than once. Nevertheless, the conserved domain of the gene was still the most frequent target of the mutation. According to data obtained about the smoking status of the studied patients, $86.7 \%$ of the patients were smokers or former smokers $(89.3 \%$ ) male and $73.3 \%$ female). It is known that the polycyclic aromatic hydrocarbons (PAHs) of tobacco smoke carcinogens primarily cause $\mathrm{G}>\mathrm{T}$ transversions, whereas nitrosamines causes $\mathrm{G}>\mathrm{A}$ 

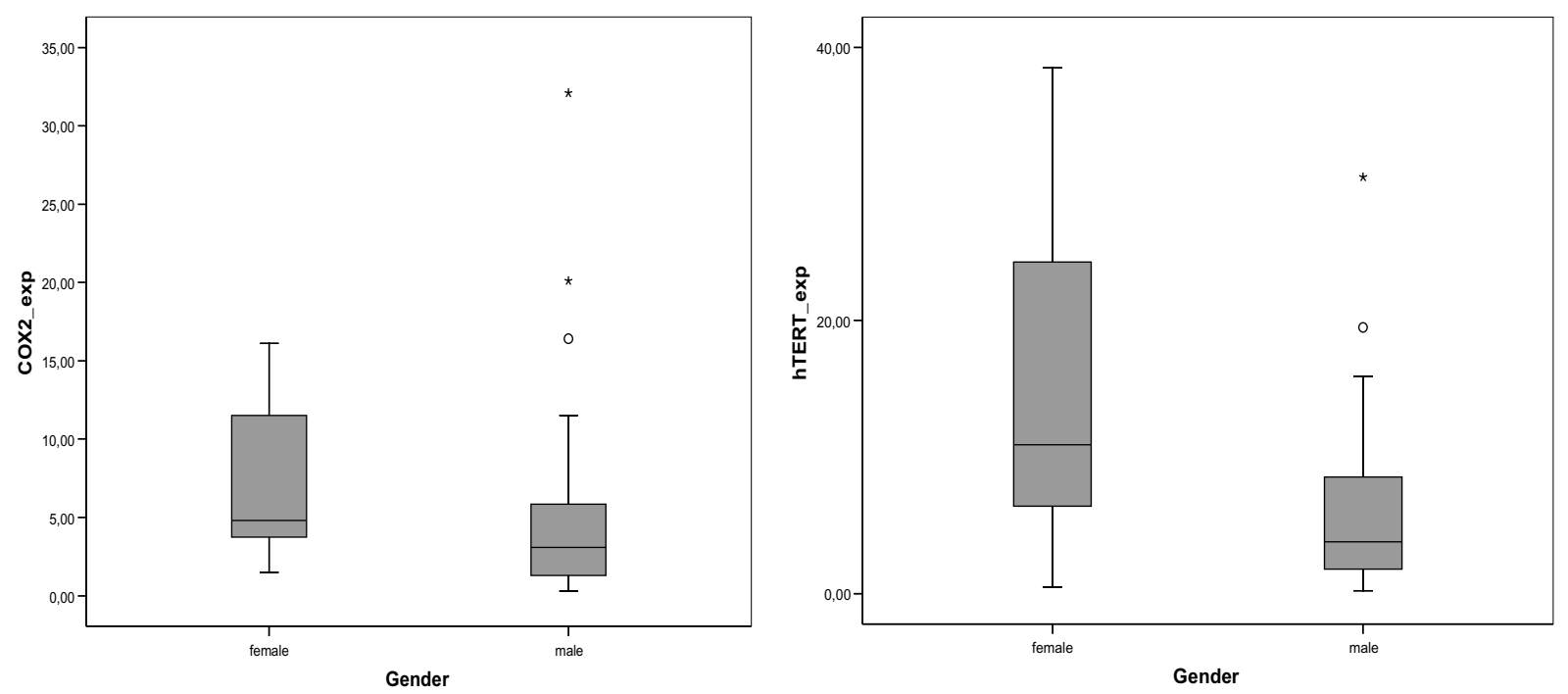

Fig. 4. Box-plot charts of the association between gender and hTERT and COX-2 expression. The associations between gender and higher expression of hTERT and COX-2 are significant for female patients $(p<0.05)$.

transitions at non $\mathrm{CpG}$ sites in the $\mathrm{P} 53$ gene $[25,26]$. We found that $55.6 \%$ of all transversions are $\mathrm{G}>\mathrm{T}$ transversions, mostly changing codons that are known as the strongest binding sites for PAHs [26]. The codons most frequently affected by mutations are V157 (11.1\% of all mutations), G245 (8.3\% of all mutations) and R283 (8.3\% of all mutations). The V157 and G245 sites are described as hotspot codons [26], but not R283. R283, found in one of every type of NSCLC studied, is not related to smoking status. The overall frequency of smoking related mutations found in P53 is in general accordance with the percentage of smokers included in our study, however, as previously established unknown exposure, geographical differences, and/or ethnicity of the patients may also influence P53 mutation spectra [25-27].

Three different mutations were discovered in the COX-2 gene, but only one (detected in two large-cell cancers) was missense. The frequency of mutations in K-RAS was significant for adenocarcinoma, which has already been recognised $[27,28]$. We found 13 mutations (all missense and mostly at codon-hotspot G12; 76.9\% of all mutations). Out of all mutations in $\mathrm{K}$-RAS, $23.1 \%$ were $\mathrm{G}>\mathrm{T}$ transversions, which is less than already published $[27,28]$. That could arise from population differences, and the fact that ADC is the type of cancer, which is related to women and never smokers.

All mutations can alter the exonic splicing enhancers (ESEs), which participate in alternative and constitutive splicing [29]. P53 has 14 significant ESE mo- tifs, according to Gorlov et al., and in our case 16 out of 36 (44.4\%) of all mutations discovered in P53 alter one of four putative significant ESE motifs [30]. ESEs are mostly altered by missense mutations $(87.5 \%$ of all ESE altering mutations), but we also discovered one deletion and one insertion in a putative ESE region. Using ESE Finder version 3.0 for prediction of ESE sites in P53 we discovered that all but 2 mutations were altering putative ESE sites [31]. The high number of mutations altering putative ESEs found was to be expected (considering that over 140 ESE motifs are in the ORF of the P53 gene and that, according to previous publication $71 \%$ of all missense mutations hit ESE sites) [30]. We also confirmed the previously published finding that missense mutations do co-localise with ESEs motifs in the P53 gene, and when analysing the K-RAS gene, we discovered that missense mutations of G12 and G13 co-localise with 2 ESEs. A putative ESE responsive to SC35 is altered by 11 out of 13 missense mutations found (84.6\%) and ESE responsive to SRp 55 by 2 out of 13 missense mutations. Missense mutations therefore strongly associate with ESE motifs (Chi square $p<0.05$ for both genes) in P53 and K-RAS genes and could give a new perspective on the functions of mutations in the coding regions of these genes. There are two possible explanations for the association; either mutations arise more frequently in ESE, or mutations in ESE are generally more pathogenic than those outside ESEs, and therefore more likely to be detected in tumours [30]. It must nevertheless be emphasized that the presence of a high-score motif in a sequence 
does not necessarily identify a functional ESE, but only predicts the possibility of one [31].

Comparison between groups with P53 and K-RAS mutations and those without revealed a strong correlation between K-RAS mutation and COX-2 expression. It was shown that COX-2 expression is regulated via the Ras signalling pathway, and induction of mutated K-RAS rapidly increases COX-2 levels [32]. A correlation of K-RAS mutations with gastric cancer, colorectal cancer and K-RAS mutated lung cancer cell lines has already been shown [32-34], but this is the first time K-RAS mutations have been linked to COX-2 expression in NSCLC tumours. We did not find any correlation between P53 mutations and COX-2 or hTERT expression. Although some publications associate P53 status with telomerase or COX-2 expression [16,18] other investigations have concluded that telomerase disorders and COX-2 activity are possibly independent of P53 mutations [22-24]; and it has already been proven that evaluation of p53 accumulation is not always comparable with P53 mutational status $[5,35,36]$.

High expression was proven not to be a consequence of staging but of gender $(p<0.05)$. The Mann-Whitney $\mathrm{U}$ test showed no correlation between gender and staging and gender was also independent of survival and mutations. Elevated expression may thus be a consequence of different pathways of tumourigenesis, different triggers for expression of COX-2 and hTERT or potential gender specific differences. It is also possible that higher expression was the result of the predominant ADC in female patients $(38.2 \%)$, but when combined with male patients the significance was lost. This is the first time that gender difference has been related to expression of COX-2 and hTERT genes.

In view of the similar clinical and biological characteristics of types of NSCLC, this study provides insight into associations among different biomarkers all commonly used in determination of the type, severity and prognosis of lung cancer. It shows that some histological and clinical features are more related to molecular genetic results than to other data, and that the use of different biomarkers combined with clinical and histological data, could result in a loss of prognostic power, although strong relations do exist between the different variables analysed.

\section{Acknowledgments}

This work was supported by Slovenian Research Agency programme P3-054.

\section{References}

[1] Y. Sekido, K.M. Fong and J.D. Minna, Molecular genetics of lung cancer, Annu Rev Med 54 (2003), 73-87.

[2] J. Yokota and T. Kohno, Molecular footprints of human lung cancer progression, Cancer Sci 95 (2004), 197-204.

[3] J. Niklinski, W. Niklinska, J. Laudanski, E. Chyczewska and L. Chyczewski, Prognostic molecular markers in non-small cell lung cancer, Lung Cancer 34 (2001), 53-58.

[4] S. Zochbauer-Muller, A.F. Gazdar and J.D. Minna, Molecular pathogenesis of lung cancer, Annu Rev Physiol 64 (2002), 681-708.

[5] Y. Tomizawa, T. Kohno, T. Fujita, M. Kiyama, R. Saito, M. Noguchi, Y. Matsuno, S. Hirohashi, N. Yamaguchi, T. Nakajima and J. Yokota, Correlation between the status of the p53 gene and survival in patients with stage I non-small cell lung carcinoma, Oncogene 18 (1999), 1007-1014.

[6] S. Rodenhuis and R.J. Slebos, Clinical significance of ras oncogene activation in human lung cancer, Cancer Res $\mathbf{5 2}$ (1992), 2665-2669.

[7] L. Wang, J.C. Soria, B.L. Kemp, D.D. Liu, L. Mao and F.R. Khuri, hTERT expression is a prognostic factor of survival in patients with stage I non-small cell lung cancer, Clin Cancer Res 8 (2002), 2883-2889.

[8] N.W. Kim, M.A. Piatyszek, K.R. Prowse, C.B. Harley, M.D. West, P.L. Ho, G.M. Coviello, W.E. Wright, S.L. Weinrich and J.W. Shay, Specific association of human telomerase activity with immortal cells and cancer, Science 266 (1994), 20112015.

[9] K. Hiyama, E. Hiyama, S. Ishioka, M. Yamakido, K. Inai, A.F. Gazdar, M.A. Piatyszek and J.W. Shay, Telomerase activity in small-cell and non-small-cell lung cancers, J Natl Cancer Inst 87 (1995), 895-902.

[10] Y. Maniwa, M. Yoshimura, C. Obayashi, M. Inaba, K. Kiyooka, M. Kanki and Y. Okita, Association of p53 gene mutation and telomerase activity in resectable non- small cell lung cancer, Chest 120 (2001), 589-594.

[11] F. Le Calvez, A. Mukeria, J.D. Hunt, O. Kelm, R.J. Hung, P. Taniere, P. Brennan, P. Boffetta, D.G. Zaridze and P. Hainaut, TP53 and KRAS mutation load and types in lung cancers in relation to tobacco smoke: distinct patterns in never, former, and current smokers, Cancer Res 65 (2005), 5076-5083.

[12] A.C. Laga, D.S. Zander, and P.T. Cagle, Prognostic significance of cyclooxygenase 2 expression in 259 cases of nonsmall cell lung cancer, Arch Pathol Lab Med 129 (2005), 1113-1117.

[13] J.E. Castelao, R.D. Bart III, C.A. DiPerna, E.M. Sievers and R.M. Bremner, Lung cancer and cyclooxygenase-2, Ann Thorac Surg 76 (2003), 1327-1335.

[14] H. Wolff, K. Saukkonen, S. Anttila, A. Karjalainen, H. Vainio and A. Ristimaki, Expression of cyclooxygenase-2 in human lung carcinoma, Cancer Res 58 (1998), 4997-5001.

[15] J. Brabender, J. Park, R. Metzger, P.M. Schneider, R.V. Lord, A.H. Holscher, K.D. Danenberg and P.V. Danenberg, Prognostic significance of cyclooxygenase 2 mRNA expression in non-small cell lung cancer, Ann Surg 235 (2002), 440-443.

[16] H. Achiwa, Y. Yatabe, T. Hida, T. Kuroishi, K. Kozaki, S. Nakamura, M. Ogawa, T. Sugiura, T. Mitsudomi and T.Takahashi, Prognostic significance of elevated cyclooxygenase 2 expression in primary, resected lung adenocarcinomas, Clin Canc Res 5 (1999), 1001-1005.

[17] J.R. Brown and R.N. DuBois, Cyclooxygenase as a target in lung cancer, Clin Canc Res 10 (2004), 4266-4269. 
[18] H. Tsubochi, N. Sato, M. Hiyama, M. Kaimori, S. Endo, Y. Sohara and T. Imai, Combined analysis of cyclooxygenase-2 expression with p53 and $\mathrm{Ki}-67$ in nonsmall cell lung cancer, Ann Thorac Surg 82 (2006), 1198-1204.

[19] E. Gross, M. Kiechle and N.J. Arnold, Mutation analysis of p53 in ovarian tumors by DHPLC, Biochem Biophys Methods 30 (2001), 73-81.

[20] E.A. König, I. Köves, A. Raşinariu, A.R. Popp, W.C. Kusser, K. Soyonki, A. Kovács, B.W. Glickman, A. Jeney and Z.L. Marcsek, Alterations of K-ras and p53 mutations in colorectal cancer patients in Central Europe, J Toxicol Environ Health A62 (2001), 333-347.

[21] K.Y. Chen, L.N. Lee, C.J. Yu, Y.C. Lee, S.H. Kuo and P.C. Yang, Elevation of telomerase activity positively correlates to poor prognosis of patients with non-small cell lung cancer, Cancer Lett 240 (2006), 148-156.

[22] C. Lu, J.C. Soria, X. Tang, X.C. Xu, L. Wang, L. Mao, R. Lotan, B. Kemp, B.N. Bekele, L. Feng, W.K. Hong and F.R. Khuri, Prognostic factors in resected stage I non-small-cell lung cancer: a multivariate analysis of six molecular markers, J Clin Oncol 22 (2004), 4575-4583.

[23] S.A. Mehdi, A.H. Tatum, N.B. Newman, G.P. Gamble, J.E. Etzell, N. Weidner, J.A. Kern, S.M. Sorscher, L.J. Kohman and S.L. Graziano, Prognostic markers in resected stage I and II non small-cell lung cancer: an analysis of 260 patients with 5 year follow-up, Clin Lung Cancer 1 (1999), 59-67.

[24] H. Sakitani, T. Tsujiuchi, K. Kobitsu, A. Kido, K. Iki, M. Takahama, M. Nakamura, T. Sakaki, D. Nakae, Y. Konishi and $\mathrm{M}$. Tsutsumi, Increased telomerase activity and absence of p53 mutation in oligoastrocytomas induced by N-ethyl-Nnitrosourea in rats, Cancer Lett 126 (1998), 157-164.

[25] P. Hainaut, M. Olivier and G.P. Pfeifer, TP53 mutation spectrum in lung cancers and mutagenic signature of components of tobacco smoke: lessons from the IARC TP53 mutation database, Mutagenesis 6 (2001), 551-553.
[26] S. Toyooka, T. Tsuda and A.F. Gazdar, The TP53 gene, tobacco exposure, and lung cancer, Hum Mutat 21 (2003), 229-239.

[27] P. Vineis and N. Caporaso, Tobacco and cancer: epidemiology and the laboratory, Environ Health Perspect 103 (1995), 156160 .

[28] K. Husgafvel-Pursiainen, P. Hackman, M. Ridanpää, S. Anttila, A. Karjalainen, T. Partanen, O. Taikina-Aho, L. Heikkilä and $\mathrm{H}$. Vainio, K-ras mutations in human adenocarcinoma of the lung: association with smoking and occupational exposure to asbestos, Int J Cancer 53 (1993), 250-256.

[29] B.J. Blencowe, Exonic splicing enhancers: mechanism of action, diversity and role in human genetic diseases, Trends Biochem Sci 25 (2000), 106-110.

[30] I.P. Gorlov, O.Y. Gorlova, M.L. Frazier and C.I. Amos, Missense mutations in cancer suppressor gene TP53 are colocalized with exonic splicing enhancers (ESEs), Mutat Res $\mathbf{5 5 4}$ (2004), 175-183.

[31] L. Cartegni, J. Wang, Z. Zhu, M.Q. Zhang and A.R. Krainer, ESEfinder: a web resource to identify exonic splicing enhancers, Nucleic Acid Research 31 (2003), 3568-3571.

[32] M.G. Backlund, J.R. Mann, D. Wang and R.N. Dubois, Ras up-regulation of cyclooxygenase-2, Methods Enzymol 407 (2005), 401-410.

[33] M. Li, W. Liu, Y.F. Zhu, Y.L. Chen, B.Z. Zhang and R. Wang, Correlation of COX-2 and K-ras expression to clinical outcome in gastric cancer, Acta Oncol 45 (2006), 1115-1119.

[34] A. Maciag, G. Sithanandam and L.M. Anderson, Mutant KrasV12 increases COX-2, peroxides and DNA damage in lung cell, Carcinogenesis 11 (2004), 2231-2237.

[35] K. Szymanska and P. Hainaut, TP53 and mutations in human cancer, Acta Biochim Pol 50 (2003), 231-238.

[36] U.M. Moll and O. Petrenko, The MDM2-p53 interaction, $M o l$ Cancer Res 1 (2003), 1001-1008. 


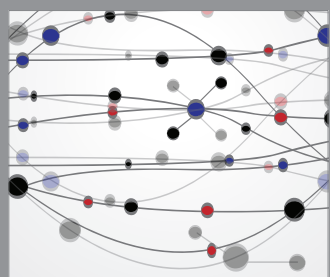

The Scientific World Journal
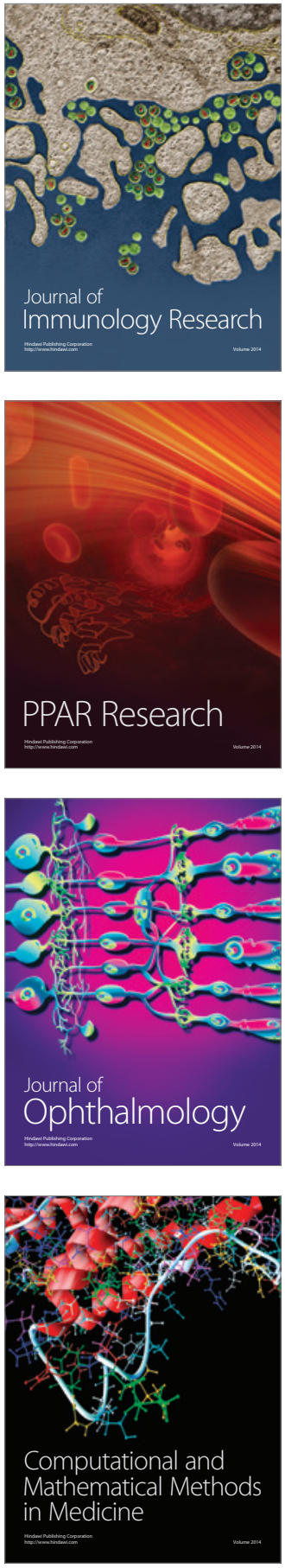

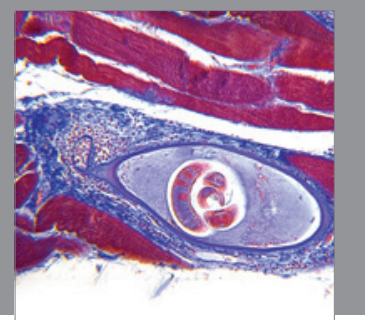

Gastroenterology

Research and Practice
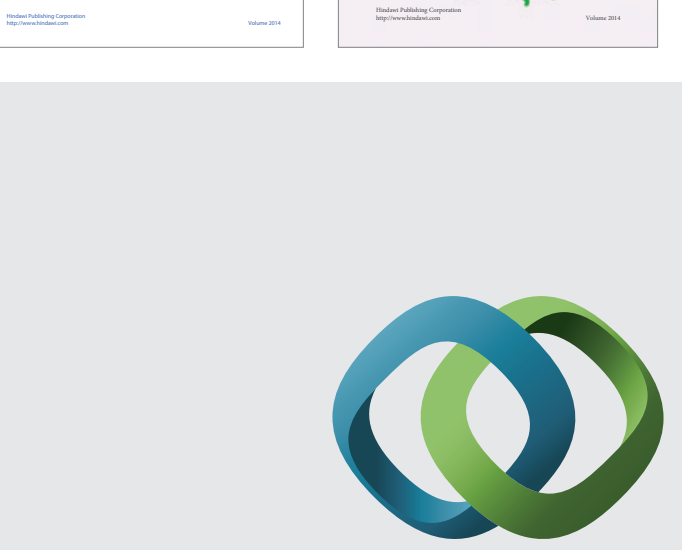

\section{Hindawi}

Submit your manuscripts at

http://www.hindawi.com
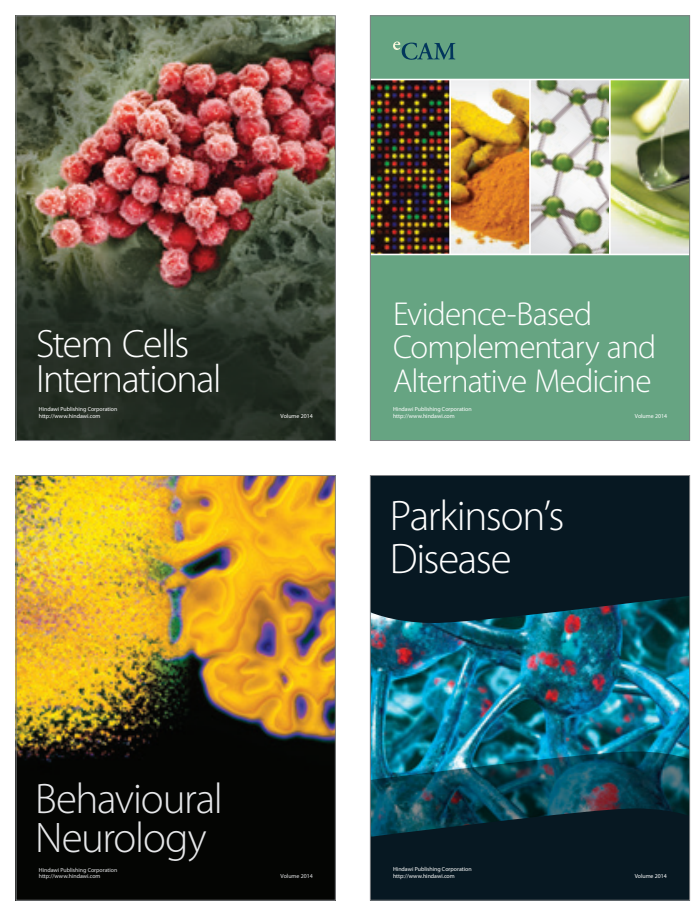

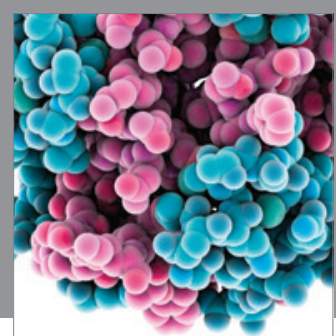

Journal of
Diabetes Research

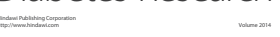

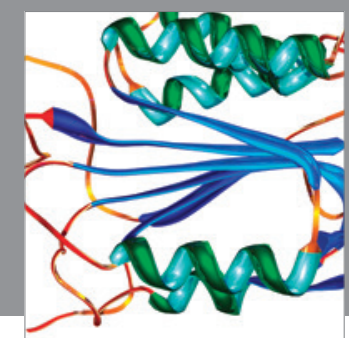

Disease Markers
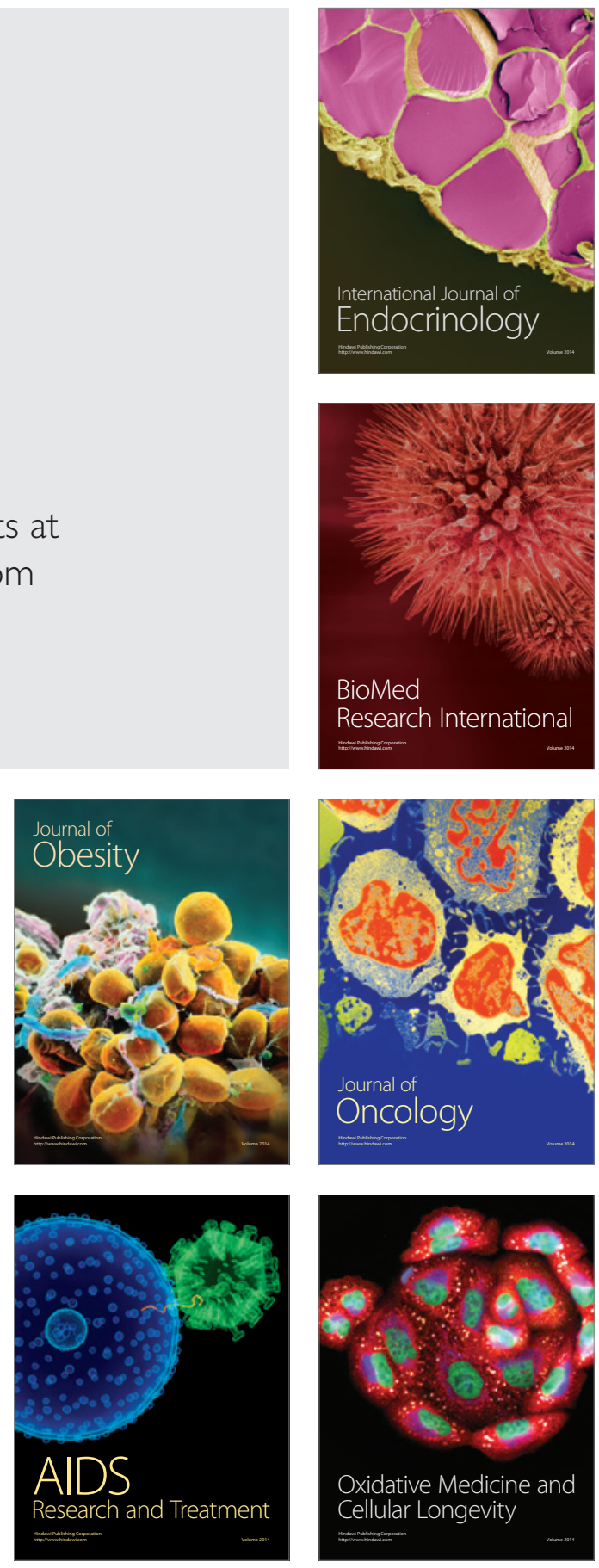